\title{
CDS -BASED ENERGY EFFICIENT TOPOLOGY CONTROL ALGORITHM IN WIRELESS SENSOR NETWORKS
}

\author{
G. N. Purohit ${ }^{1}$, Seema Verma ${ }^{2}$, Usha Sharma ${ }^{3}$, Gargi Chauhan ${ }^{4}$ \\ ${ }^{I}$ Depatment of Mathematics and Statistics, Banasthali University, Banasthali, Rajasthan-304022, India \\ ${ }^{2}$ Department of Electronics, Banasthali University, Banasthali, Rajasthan-304022, India \\ ${ }^{3}$ Depatment of Mathematics and Statistics, Banasthali University, Banasthali, Rajasthan-304022, India \\ ${ }^{4}$ Department of Computers, Banasthali University, Banasthali, Rajasthan-304022, India
}

\begin{abstract}
Wireless Sensor Networks (WSNs) are a self organized network which consists of large number of sensor nodes that collects the data in a various environment [1,2]. The sensors work on battery that have limited lifetime so it is a challenge to create an energy efficient network that can reduce the energy consumption and interference in the network graph and thereby extend the network lifetime [2]. For saving energy and extending network lifetime the topology is a well-known technique in WSNs and the widely used topology control strategy is the construction of Connected Dominating Set (CDS) [3, 4]. In this paper, we construct a CDS based energy efficient topology control algorithm i.e. GCDSTC for WSNs. The performance analysis includes the study of GCDSTC algorithm in terms of complexity and compares it with EBTC (Energy Balanced Topology Control) algorithm. The simulation results indicate that the GCDSTC algorithm reduce the energy consumption and interference in the network graph, in order to enhance the network lifetime.
\end{abstract}

Keywords: Wireless Sensor Network (WSN), Connected Dominating Set (CDS), Topology Control (TC), etc.

\section{INTRODUCTION}

Wireless Sensor Networks (WSNs) consists of a large number of sensor nodes which is low-power, inexpensive and unobtrusive [5]. It has been used to collect reliable and accurate information in the distance and hazardous environment [2]. And also it has been considered in civilian application areas such as environmental monitoring, traffic monitoring, disaster management, health monitoring and military applications $[2,3]$.

In WSNs, the sensor node is a tiny device which is small in size and has limited battery power and communication capabilities due to which a node can cover only those sensor nodes that have been occurred within its maximum transmission range [2, 6]. Sensor nodes can gather information from source, process it and transmit it to the sink node or source node [6]. The sensor node has been run on batteries so that it has been quite difficult to exchange or recharge node batteries [5, 6]. The lifetime of the sensor node depends on the lifetime of power resources so that the improvement of energy efficiency and power management is a critical issue in the WSNs [5].

Due to this problem, a virtual backbone in WSNs has been created through which each node of the network can transmitted data to the sink node effectively. A virtual backbone is a set of active nodes through which the data has been transmitted to the sink node by forwarding data to other neighbouring active nodes [2].
In WSNs, the Connected Dominating Set (CDS) based Topology Control (TC) is the energy efficient method [2]. Topology Control is one of the known strategies to save energy or to control energy consumption in WSNs [3, 4]. The topology control has been divided into two components: topology construction (reduced topology or change of the initial topology) and topology maintenance (maintenance of the reduced topology) while ensuring the connectivity and coverage $[2,3,7]$. In CDS-based TC, the nodes in the CDS are called dominators or active nodes, and the other nodes which are not in the CDS are called dominatees or sleep node. Therefore, CDS works as a virtual backbone in the WSNs.

The main objective of this paper is to have a network which has minimum energy consumption and interference and also has maximum network lifetime. In this paper GCDSTC algorithm in a directed graph has been constructed, models the topology as a connected network and finds the set of active nodes which form a CDS. The network has been considered as a graph where the vertices correspond to the nodes and the edges correspond to the links between these vertices or nodes. One node is considered as the access point (AP). All the data has been transferred from each node should reach the AP.

The active nodes and sleep nodes of the network graph have been found according to the degree and energy of the nodes and through the active nodes the data has been transmitted to the AP. These active nodes are also called the dominators. For implementing this process, GCDSTC algorithm has been developed which is based on the above approach. 
Through the proposed algorithm the links in the network graph has been reduced due to which the energy consumption and interference is minimized. In this way the lifetime of the network can be extended.

The GCDSTC algorithm has been evaluated using simulations and compared with the EBTC algorithm [13]. In terms of complexity the result shows that the GCDSTC algorithm performs better than EBTC (Energy Balanced Topology Control) algorithm.

The rest of the paper is organized as follows. Section 2 reviews the related works. Section 3 describes the system model. Section 4 presents problem statement. Section 5 presents MICDS algorithm. Section 6 describes and analyzes the simulation results for the proposed algorithm. Section 7 presents the performance evaluation. Section 8 concludes the paper.

\section{RELATED WORK}

To solve energy consumption problem in WSNs, many topology control techniques have been proposed. $\mathrm{Ya} \mathrm{Xu}$ et al. (2003) have presented two topology control protocols that prolong dense ad hoc networks lifetime while maintaining connectivity, the ability for nodes to reach each other. By identifying redundant nodes their protocols conserve energy and turning off their radios. In WSNs the topology control with per-node transmission power adjustment has been shown to be effective with respect to prolonging the lifetime of the network through power conservation and increasing network capacity through better spatial bandwidth reuse [8]. A simple yet efficient distributed topology control algorithm has been proposed in Jilei Liu and Baochun Li (2003). To compute per-node minimum transmission power a distributed topology control algorithm has been presented. To control topology process for application nodes (AN) and base station (BS), the twotiered WSN is good initiative which usually resides in the upper layer [9]. Jianping Pan et al. (2003) have proposed an approach which maximizes the lifetime of the topological network of the WSN by arranging BS location and inter-AN relaying optimally. By using the computational geometry, derived the optimal BS locations for mission criticality and the efficiency of topology control validates the optimality [10].

Topology control in heterogeneous WSN is more complex. In Chun-Hsien Wu and Yeh-Ching Chung (2007), a heterogeneous WSN deployment method based on irregular sensor model has been proposed. The aim of this method is to deal with the deployment problem of heterogeneous sensor nodes with different communication and sensing range. To approximate the behaviour of sensor nodes the irregular sensor model has been proposed. The proposed method can achieve higher coverage rate for the same deployable sensor nodes [11].

Adopting the transmission power in WSNs is critical to determine the performance of the network in terms of energy efficiency, connectivity and spatial reuse since it has direct impact on the physical network topology. C. Paolo (2009) has proposed a solution i.e. cooperative and light weight to the problem of controlling the local connectivity in WSNs. Evaluate the topology control solution accurately; a small-scale testbed based on Micaz sensor nodes is deployed in indoor and outdoor scenarios. The solutions provides the steady network connectivity while reducing the overall power consumption [12].

A3 algorithm which is simple distributed and energy efficient topology construction mechanism have been proposed by Pedro M. Wightman and Miguel A. Labrador (2008) that produces an approximate solution to form a suboptimal CDS. To allow the communication between any arbitrary pair of nodes in the network a virtual backbone is formed in a CDS-based topology control technique [3]. A.Karthikyan et al. (2013) have proposed a CDS based topology control algorithm TCI which forms an energy efficient virtual backbone. TCI achieves better connectivity under topology maintenance and also provides better sensing coverage [2].

\section{SYSTEM MODEL AND NETWORK MODEL}

The WSN is represented by a graph $G=(V, E)$, where $V=\left\{\mathrm{v}_{1}, \mathrm{v}_{2}, \mathrm{v}_{3}, \ldots, \mathrm{v}_{\mathrm{n}}\right\}$ denotes the set of all nodes in the graph, including the access point (AP) and $\mathrm{E} \subseteq v \times v$ denotes the set of all edges referred to all the communication links through which the data packets can be transmitted from one node to another. All the communication links have been destined to AP. Every data packet from a node has been forwarded to its parent node so that the entire data packet finally reached the AP.

If $\left\{\mathrm{v}_{\mathrm{i}}, \mathrm{v}_{\mathrm{j}}\right\} \subseteq V$, the edge $e=\left\{\mathrm{v}_{\mathrm{i}}, \mathrm{v}_{\mathrm{j}}\right\} \in E$ if and only if ' $\mathrm{v}_{\mathrm{j}}$ ' is located within the transmission range of ' $v_{i}$ '. In a directed graph, the edge ' $\mathrm{e}$ ' is called incident from ' $\mathrm{v}_{\mathrm{i}}$ ', and incident to ' $v_{j}$ ', but in an undirected graph, the edge ' $e$ ' may be incident to or from ' $v_{\mathrm{i}}$ ' and ' $\mathrm{v}_{\mathrm{j}}$ '. In graph, each node ' $\mathrm{v}$ ' can communicated with a subset $N(v) \subseteq V$ of nodes, where each node $u \in N(v)$ is called the neighbor of ' $v$ '.

Topology control is one of the techniques that control energy consumption in WSNs and also increase the lifetime of the network. It is the management of network topology to support the network requirements. In the reduced constructed topology the CDS works as a virtual backbone.

\section{PROBLEM STATEMENT}

In WSNs, a virtual backbone is a connected dominating set (CDS). And CDS based topology control is the method for energy efficient in WSNs. This method has been used to reduce the energy consumption and interference in network graph and also used to extend the network lifetime.

GCDSTC algorithm has been constructed which is based on the CDS based topology control and it has been used to solve the energy consumption and interference problem in the directed network graph. In this algorithm the active nodes and sleep nodes of the network have been found and 
also reduce the number of active nodes and active links. Through these active nodes the data has been transmitted to the sink node in the network. So that GCDSTC algorithm provides the network with less energy consumption and interference and also provides the increment in the network lifetime.

\section{GCDSTC ALGORITHM}

The algorithm constructs the topology which is based on the CDS- based topology control. It will provide the transmission of the data to the sink with less energy consumption and interference due to which the network lifetime has been extended.

Step 1: Arrange all the vertices of the network graph ' $G$ ' in ascending order.

Step 2: Find the out_degree and in_degree of the vertices of the network graph.

Step 3: If the out_degree $\left(\mathrm{v}_{\mathrm{i}}\right)==0$ then

$$
\mathrm{v}_{2} \rightarrow \text { sink node }(\mathrm{SN})
$$

Put sink node (SN) in active state (AS)

If the in_degree $\left(\mathrm{v}_{\mathrm{i}}\right)==0$ then

$\mathrm{v}_{\mathrm{i}} \rightarrow$ Leaf Node (LN)

Put leaf node (LN) in sleep state (SS)

Step 4: Find the neighbor vertices of the sink node

$\mathrm{N}_{\mathrm{sn}} \rightarrow \mathrm{v}_{\mathrm{i}}, \mathrm{v}_{\mathrm{i}+1}, \ldots \ldots, \mathrm{v}_{\mathrm{n}}$

Put the neighboring vertices of the sink node $\left(\mathrm{N}_{\mathrm{sn}}\right)$ in active state (AS)

$$
\begin{gathered}
\text { If } \mathrm{N}_{\text {sni }}==\mathrm{LN} \text { then } \\
\mathrm{N}_{\mathrm{sni}} \rightarrow \mathrm{SS}
\end{gathered}
$$

Step5: Remove a sink node, leaf node and neighboring node of sink node from the vertex set ' $\mathrm{V}$ ' of the network graph 'G'.

$$
\mathrm{V}^{\prime}=\left[\mathrm{V}-\left(\mathrm{SN}+\mathrm{LN}+\mathrm{N}_{\mathrm{sn}}\right)\right]
$$

Step 6: Find the energy of the $V^{\prime}$ ' vertices of the network graph ' $\mathrm{G}$ '

$$
\mathrm{V}_{\text {eng }}=\mathrm{v}_{1 \mathrm{e}}, \mathrm{v}_{2 \mathrm{e}}, \ldots \ldots . ., \mathrm{v}_{\mathrm{ne}}
$$

Step 7: Arrange the energy and degree of all the vertices of the vertex set $V$ ' of the network graph ' $G$ ' in descending order.

Calculate

$$
\begin{aligned}
& \text { If } v_{i}(E)>v_{i+1}(E) \text { then } \\
& \mathrm{v}_{\mathrm{i}}(\mathrm{E}) \rightarrow \mathrm{EN}_{\mathrm{i}} \\
& \mathrm{v}_{\mathrm{i}}(\mathrm{D}) \rightarrow \mathrm{Deg}_{\mathrm{i}} \\
& \text { else } \\
& \mathrm{v}_{\mathrm{i}+1}(\mathrm{E}) \rightarrow \mathrm{EN}_{\mathrm{i}} \\
& \mathrm{v}_{\mathrm{i}+1}(\mathrm{D}) \rightarrow \operatorname{Deg}_{\mathrm{i}} \\
& \text { If } v_{i}(E)==v_{i+1}(E) \text { then } \\
& \text { If } v_{i}(D)>v_{i+1} \text { (D) then } \\
& \mathrm{v}_{\mathrm{i}}(\mathrm{E}) \rightarrow \mathrm{EN}_{\mathrm{i}} \\
& \mathrm{v}_{\mathrm{i}}(\mathrm{D}) \rightarrow \operatorname{Deg}_{\mathrm{i}} \\
& \text { else } \\
& \mathrm{v}_{\mathrm{i}+1}(\mathrm{E}) \rightarrow \mathrm{EN}_{\mathrm{i}} \\
& \mathrm{v}_{\mathrm{i}+1}(\mathrm{D}) \rightarrow \operatorname{Deg}_{\mathrm{i}}
\end{aligned}
$$

Step 8: After finding the energy and degree of the vertices of the network graph ' $G$ ' in some order. The user selects the vertices in active state (AS) and sleeps state (SS) according to its requirements for the transmission. Put the active state vertices in connected dominating set (CDS) as dominators and the sleep state vertices as dominatees.

Thus, the topology of the network graph that has minimum number of CDS have been obtained.

\section{SIMULATION RESULTS}

In this section, the performance of GCDSTC algorithm has been analyzed by simulation in MATLAB version R2007a. Our evaluations are based on the finding of active nodes and sleep nodes of the network graph. Using active nodes, the minimum number of the CDS is found out. Through the CDS we can transmit all the data to the sink node. And this can also reduce the energy wastage and collisions or interference between the nodes of the graph and extend the lifetime of the network.

The following procedures have been simulated on MATLAB for finding the active nodes and sleep nodes in the network graph:

\subsection{Procedure GCDSTC}

Input: $\mathrm{Graph} \mathrm{G}=(\mathrm{V}, \mathrm{E})$

' $n$ ' is the total number of vertices in the graph

'total' is the degree of the vertices of the graph

'AS' is the active state nodes in the graph

' $\mathrm{SS}$ ' is the sleep state nodes in the graph

'in_deg' is the indegree of the vertex

'out_deg' is the outdegree of the vertex

For all the vertices ' $\mathrm{V}$ ' of the graph stored at ' $\mathrm{i}$ ' do

Enter the indegree of vertices of ' $i$ ' stored at 'in_deg'

Enter the outdegree of vertices of ' $i$ ' stored at 'out_deg'

Check if out_degree is zero then

Sink node is ' $i$ ' whose outdegree is zero

Put that 'sink node' in the AS

End

Check if in_degree is zero then

Leaf node is ' $\mathrm{i}$ ' whose in_degree is zero

Put that 'leaf node' in the SS

End

Calculate total degree of the all the vertices

End

For all the vertices ' $\mathrm{V}$ ' of the graph stored at 'i' do

Check if ' $i$ ' is sink node then

Enter the adjacent vertices of ' $i$ ' stored at 'Nsink' End

End

Put the 'Nsink' in AS

Remove sink node, leaf node and Nsink from the ' $\mathrm{V}$ '

$\mathrm{V}^{\prime}$ is the remaining vertices after the removal of sink node, leaf node and Nsink

$\mathrm{V}^{\prime}=\mathrm{V}-($ sink node + leaf node $+\mathrm{N}$ sink $)$

For all the vertices ' $\mathrm{V}$ ' of the graph stored at 'i' do

Check if $\mathrm{i} \sim=\mathrm{V}^{\prime}$

Enter the energy of vertices of ' $i$ ' stored at 'ener' 
Find the total energy and degree of each of vertices in $V^{\prime}$ and store that value in 'ee' and ' $d$ '.

Sort the 'ee' and 'd' value in descending order and store that value in 'eg' and 'deg'.

User selects the vertices from 'eg' according to its requirement. And put that vertices in $\mathrm{AS}$ and the remaining vertices in SS.

Therefore, in the above procedure 'AS' and ' $\mathrm{SS}$ ' is the answer. And the active state and sleep state have been represented in the form of matrix.

\subsection{Analysis}

We have analyzed that the transmission of the data in the network graph using CDS based topology control methods depends on the active state nodes of the network graph.

Table 1 Active state nodes of the network graph

\begin{tabular}{|c|c|c|c|}
\hline $\begin{array}{c}\text { Number of } \\
\text { vertices }\end{array}$ & $\begin{array}{c}\text { Number of } \\
\text { edges in } \\
\text { graph }\end{array}$ & $\begin{array}{c}\text { Number of } \\
\text { edges in } \\
\text { topology }\end{array}$ & $\begin{array}{c}\text { Active } \\
\text { State } \\
\text { Nodes }\end{array}$ \\
\hline 11 & 18 & 10 & 7 \\
\hline 20 & 30 & 19 & 10 \\
\hline 25 & 38 & 24 & 18 \\
\hline 30 & 46 & 29 & 19 \\
\hline 40 & 71 & 39 & 21 \\
\hline
\end{tabular}

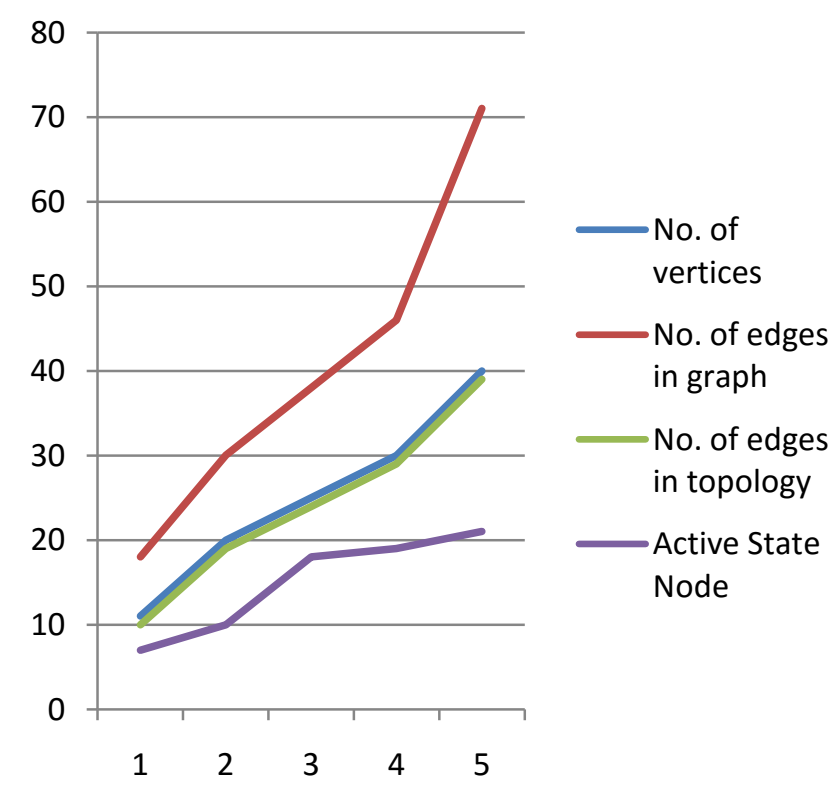

Fig 1 Simulation results of the algorithm

From Table 1 and Figure 1, the four cases have been analyzed:

1. Minimum number of edges in the topology of the network is equal to the (n-1) vertices.

2. Topology of the network graph has minimum number of vertices in the active state which means the network graph has minimum number of connected dominating set (CDS).
3. Maximum transmission through each nodes is (n-SN$\mathrm{LN}-\mathrm{N}$ sink) where ' $\mathrm{n}$ ' is the number of vertices, ' $\mathrm{SN}$ ' is the sink node of the network graph, ' $\mathrm{LN}$ ' is the leaf node of the network graph and 'Nsink' is the neighbor nodes of the sink node in the network graph.

4. Minimum vertices in active state are $(\mathrm{SN}+\mathrm{N}$ sink $)$.

\section{PERFORMANCE EVALUATION}

In this section, we have been compared our algorithm i.e. GCDSTC algorithm with EBTC algorithm in terms of time complexity [13]. In our algorithm, the time complexity of the algorithm is $\mathrm{O}(\mathrm{n})$. On the other hand, the overall complexity of the EBTC (Energy Balanced Topology Control) algorithm is $\mathrm{O}\left(\mathrm{n}^{2}\right)$ in terms of time, where ' $\mathrm{n}$ ' is the total number of nodes in the network. Clearly, our algorithm has less time complexity than EBTC algorithm for running the whole process.

\section{CONCLUSION}

In this paper, a new topology control method has been proposed which is based on CDS-based topology control method that is GCDSTC algorithm. The algorithm finds the active state nodes of the network graph that has been created the minimum CDS of the network graph. Therefore, the CDS or active state nodes have been used for the transmission of the data packets to another node or sink node. The resulting assignment can guarantee that there is no interference between the nodes due to which the consumption of energy is decreased and the lifetime of the network is increased. The complexity of the GCDSTC algorithm has been calculated which is $\mathrm{O}(\mathrm{n})$ and compared it with EBTC (Energy Balanced Topology Control) algorithm and find that the GCDSTC algorithm is better than the EBTC algorithm.

\section{ACKNOWLEDGMENTS}

One of the authors of this paper, Gargi Chauhan acknowledges UGC, New Delhi for the financial assistance for carrying out this research.

\section{REFERENCES}

[1]. Jian Ma, Chen Qian and Qian Zhang, “Opportunistic Transmission based QoS Topology Control in Wireless Sensor Networks",IEEE, 2008, pp 1-6.

[2]. A. Karthikeyan, T. Shankar, V. Srividhya, Siva Charan Reddy. V and Sandeep Kommineni, "Topology Control Algorithm for Better Sensing Coverage with Connectivity in Wireless Sensor Networks", Journal of Theoretical and Applied Information Technology, Vol. 52 No.3, 30th June 2013.

[3]. Pedro M. Wightman and Miguel A. Labrador, “ $A 3$ : $A$ Topology Construction Algorithm for Wireless Sensor Networks", GLOBECOM 2008: 346-351.

[4]. Parikha Chawla, Parmender Singh and Taruna Sikka, "Enhance Throughput in Wireless Sensor Network Using Topology Control Approach”, International Journal of Soft 
Computing and Engineering (IJSCE) ISSN: 2231-2307, Volume-2, Issue-3, July 2012.

[5]. Alexis Papadimitriou, Dimitrios Katsaros and Yannis Manolopoulos , "Topology control algorithms for wireless sensor networks: A critical survey”, CompSysTech 2010: 110.

[6]. Ramakant Sharma and Praveen Kaushik, "Network Coding Based Virtual Backbone for Wireless Sensor Network", International Journal of Computer Science and Information Technologies (IJCSIT), Vol. 3 (3), 2012,4341 4344.

[7]. G.N. Purohit and Usha Sharma, "Topology Control for Energy Conservation in Wireless Sensor Network", Int. J. Contemp. Math. Sciences, Vol. 7, 2012, no. 2, 53 - 6.

[8]. Ya Xu, S. Bien, Y. Mori, J. Heidemann and D. Estrin, "Topology Control Protocols to Conserve Energy in Wireless Ad Hoc Networks", CENS Technical Report, 2003.

[9]. Jilei Liu and Baochun Li, "Distributed Topology Control in Wireless Sensor Networks with Asymmetric Links”, GLOBECOM 2003 - IEEE Global Telecommunications Conference, no. 1, pp. 1257-1262, Dec 2003.

[10]. Jianping Pan, Y. Thomas Hou, Lin Cai, Yi Shi and Sherman X. Shen, "Topology control for wireless sensor networks" , ACM on Mobile Computing and Networking, pp. 286-299, Sep. 2003.

[11]. Chun-Hsien $\mathrm{Wu}$ and Yeh-Ching Chung, "Heterogeneous Wireless Sensor Network Deployment and Topology Control Based on Irregular Sensor Model", Advances in Grid and Pervasive Computing Lecture Notes in Computer Science, Volume 4459/2007, 2007.

[12]. C. Paolo, C. Matteo, B. Stefano, and C. Luca, "A Cooperative Approach for Topology Control in Wireless Sensor Networks", Pervasive and Mobile Computing, vol. 5, no. 5, 2009, pp. 526-541.

[13]. X. Chu and H. Sethu , “An Energy Balanced Dynamic Topology Control Algorithm for Improved Network Lifetime", Computer Science Networking and Internet Architecture 12 sep 2013. 